\title{
Digital Depth of Field Control in Photography based on Focused Plenoptic Camera
}

\author{
Peng Liü ${ }^{1,2, a}$, Rumin Zhang ${ }^{1,2, ~ b}$, Dijun Liü, c \\ ${ }^{1}$ School of Electronic and Information Engineering, Beijing University of Aeronautics \& Astronautics, \\ No.37 Xueyuan Road, Haidian Distract,Beijing,China \\ ${ }^{2}$ State Key Laboratory of Wireless Mobile Communications, China Academy of \\ Telecommunications Technology (CATT),Beijing,China \\ aemail: 715248894@qq.com, bemail:rm_zhang@126.com, cliudijun@leadcoretech.com
}

Keywords: Focused Plenoptic Camera; Digital Refocusing; Depth of Field Control; Micro Len Array

\begin{abstract}
When the exposure is over, the focused plane and depth of field(DOF) is definite. It is necessary for us to properly adjust the aperture size, exposure time and focus before we take photos. The paper deduces a patch-to-patch reconstruction algorithm from the structure of focused plenoptic camera proposed by Georgiev and Lumsdaine. It can not only build a high resolution refocus image, but supply us with the capability of DOF control after the exposure, just like we take photos under different aperture sizes. With the algorithm, we can always shoot picture under the maximum aperture size to maintain the best SNR result, and render the image taken at desired focused plane and DOF from the light field data of the scene on computer. And the simulation result indicates that the proposed algorithm is feasible and effective.
\end{abstract}

\section{Introduction}

The depth of field(DOF) can be seen as a continuous range within which the objects in the scene are in focus. When we increase the amount of light through the lens by using large aperture, it will get the result picture of high SNR, shallow DOF and a fast shutter speed. Shallow DOF is very useful when we want to highlight the subject in the photo, while large DOF will make more objects we are interested in sharp and clear. But large DOF needs small aperture size and a long exposure time. And it may bring a low SNR image.

So traditional photography needs us to compromise the aperture size, DOF and exposure time before we press the shutter. Because they cannot be changed when the exposure is over, they should be correctly set or it will lead to a shooting failure. It is desirable if we always use large aperture to shot the scene in short exposure time, and render a picture with arbitrarily specified focus and DOF to meet the practical demand. Now the plenoptic camera appears to solve the problem.

The first plenoptic camera was put forward by Adelson and Wang[1]. And it is later improved by $\mathrm{Ng}[2]$ and implemented in a hand held 645 camera. The propose of digital refocusing enables us to change focus when the exposure is over. Focused plenoptic camera is a later structure optimized by Georgiev and Lumsdaine[3]. It sacrifices directional resolution to get higher spatial resolution in return. Based on the focused plenoptic camera, rendering a high resolution refocusing image is much easier to meet the requirement of modern photography.

The paper first takes a brief review of the feature of focused plenoptic camera. And from the its structure it deduces a high resolution digital refocus algorithm. The algorithm introduces refocus and aperture factors and enables us to change focus of rendered image from a single-exposure plenoptic data. Besides, it gives us the capability of changing from shallow DOF to all-in-focus image, realize DOF control after exposure. Using the algorithm, high resolution images of different focus and DOF are rebuilt from the radiance samples captured on the sensor. From the simulation result, it can be learnt that the algorithm is feasible and meet the needs of modern photography. 


\section{Structure of Focused Plenoptic Camera}

Fig. 1 demonstrates the structure of focused plenoptic camera proposed by Georgiev and Lumsdaine. It is composed of a main lens and a micro lens array inserted in front of the image sensor. Generally, the micro lenses in the array are arranged in matrix style.

The array of micro lenses inserted between the main lens and the sensor has a distance $v$ in front of the sensor plane. They project the image created by the main lens onto the sensor. Their image circles divide the sensor into sub images. The position of sub images $(m, n)$ combining with the pixels in sub images $(s, t)$ give us the radiance corresponding to a definite direction and position. They form the 4D light field $I(s, t, m, n)$. In a practical plenoptic system, we read the 4D light field data $I(s, t, m, n)$ from the sensor. Consider the sensor data has the $2 \mathrm{D}$ form of $I_{s}(x, y)$. From the Fig. 2, we can see the relationship between a light ray of definite direction and position and pixel on the sensor. Thus the light field $I(s, t, m, n)$ can be derived from the sensor date $I_{s}$ :

$$
I(s, t, m, n)=I_{s}\left(m^{*} N x+s, n * N y+t\right)
$$

where $N x^{*} N y$ is the size of each sub image.

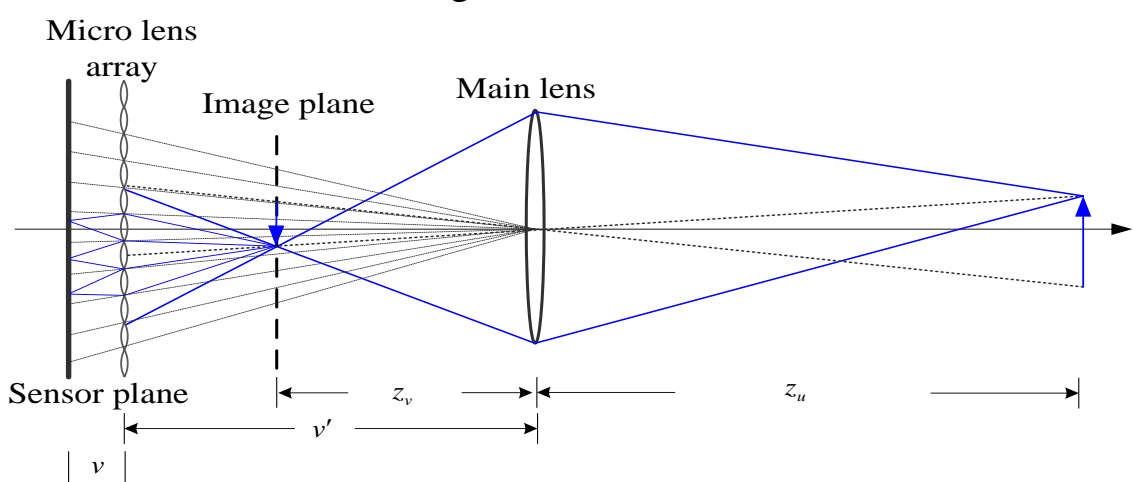

Fig.1: Structure of focused plenoptic camera

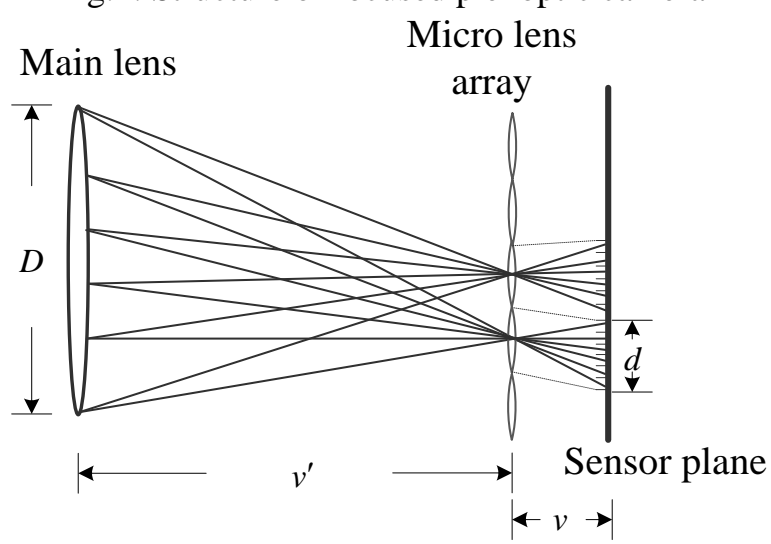

Fig.2: Sampling of plenoptic camera

\section{Digital Depth of Field Control Algorithm}

According to the structure of focused plenoptic camera introduced in the previous section we propose a rendering algorithm to get an output image at specified focus plane and aperture size from reconstructed $4 \mathrm{D}$ light field $I(s, t, m, n)$ in (1).

Suppose that the refocus plane has a distance of $\alpha v^{\prime}$ to the main lens, where $\alpha$ is the refocus factor that decides the position of refocus plane. Small $\alpha$ means the rendered image refocus to a further position, while large $\alpha$ indicates the rendered image refocus to the near position. Suppose the number of micro lenses in the array is $N X * N Y$. It is known in Fig. 3 that the connection lines between the optic center of the main lens and the edge of the micro lenses divide the refocus plane into $N X * N Y$ patches, the same as the number of micro lenses. The rendering process will be 
taken from patch to patch. Before that we take an arbitrary refocused pixel $\left(s_{\alpha}, t_{\alpha}\right)$ on the reconstructed plane for example to illustrate how the plenoptic data on the sensor contributes to refocus plane.

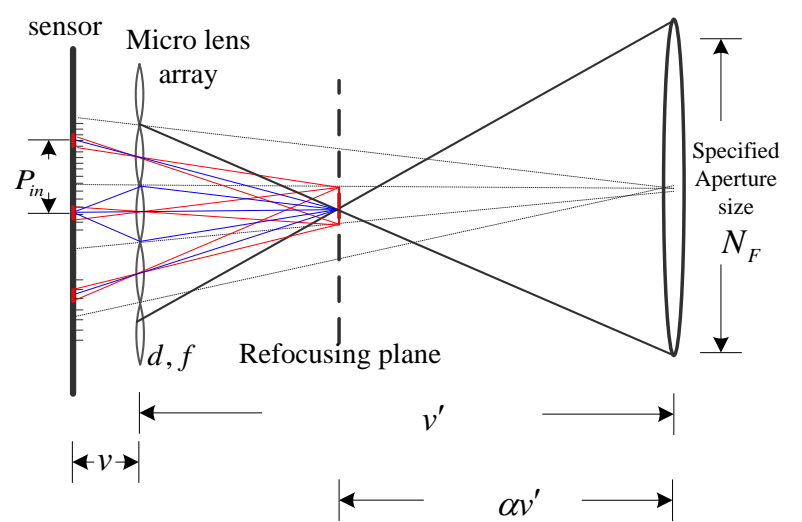

Fig.3: Digital refocusing algorithm with DOF control

When the refocus factor $\alpha$ is fixed, the light projection of an arbitrary point $\left(s_{\alpha}, t_{\alpha}\right)$ to the micro lens array overlays a certain number of micro lenses, whose sub images offer effective information to the refocus point. And it can be known that the diameter of this projection from $\left(s_{\alpha}, t_{\alpha}\right)$ is decided by the specified photographing aperture size $N_{F}$ and refocus factor $\alpha$ of the reconstructed image. The diameter(number of micro lenses) can be calculated:

$$
L=\left[\frac{1-\alpha}{\alpha} \cdot \frac{F / N_{F}}{d}\right]
$$

[ ] is the rounding symbol. Sub images of the micro lenses that lay within this range have effective information of $\left(s_{\alpha}, t_{\alpha}\right)$. We consider them as the effective sub images of $\left(s_{\alpha}, t_{\alpha}\right)$.

The radiance of $\left(s_{\alpha}, t_{\alpha}\right)$ is the weighted average of all the corresponding pixels of $\left(s_{\alpha}, t_{\alpha}\right)$ on the sensor(Blue line on the sensor in Fig. 3). Under the assumption that the objects in the scene are Lambertian. So they produce redundant radiance in different angles. We can figure that all corresponding pixels of $\left(s_{\alpha}, t_{\alpha}\right)$ on the sensor contribute the same to the radiance of $\left(s_{\alpha}, t_{\alpha}\right)$. Thus all weights are equal. Here we consider that $1 / W$ denotes the weight, where $W$ is the number of effective pixels in all sub images.

The output image is rendered patch by patch. For an arbitrary patch $I_{\alpha}\left(s_{\alpha}, t_{\alpha}, m, n\right)$, the number of its effective pixels in its effective sub images is(red line part on the sensor in Fig.3):

$$
P_{\text {in }}=\left[\frac{\alpha}{1-\alpha} \cdot \frac{v}{v^{\prime}} \cdot \frac{d}{\delta}\right]
$$

Thus $P_{i n}$ is a function of position factor $\alpha$.

Under a specified rendering aperture and refocus factor, only those sub images that can "see" the sector $I_{\alpha}\left(s_{\alpha}, t_{\alpha}, m, n\right)$ donates $P_{i n} * P_{i n}$ pixels to this sector. It has:

$$
\begin{aligned}
I_{\alpha}\left(s_{\alpha}, t_{\alpha}, m, n\right) & =\frac{1}{W} \sum_{k} \sum_{l} I\left(\frac{N x+P_{i n}}{2}-i_{m}+k^{*} P_{i n}, \frac{N y+P_{i n}}{2}-i_{m}+l * P_{i n}, m+k, n+l\right), \\
0 \leq m & <N X, 0 \leq n<N Y
\end{aligned}
$$

where $K$ is the total number of items in the sum. The range of $k, l$ is discussed here. We define $\operatorname{Cen}(m, n)$ as the center position coordinates of the $m, n$-th micro lens. For all pair of the $k, l$ if one

satisfy: $\quad(\operatorname{Cen}(m+k, n+l)-\operatorname{Cen}(m, n))^{2} \leq\left(\frac{1-\alpha}{\alpha} \cdot \frac{F / N_{F}}{d}\right)^{2}$

then the $k, l$-th sub image will be conducing in the final sum. The final output is the joint image of all patches. The resolution of the reconstruction image is $P_{\text {in }} * P_{i n} * N X * N Y$.

In the refocus algorithm with DOF controlling, large $N_{F}$ means rendering with small aperture 
and large DOF. Especially when the $N_{F}$ reaches its maximum, only one effective sub image can "see" the rendering patch. The rendered result is all-in-focus from the front to the background.

\section{Test results}

In this section, we render results from a pre-processed plenoptic data to illustrate the feasibility and effectiveness of the algorithm. This picture was shot by T.Georgie with the prototype of focused plenoptic camera, as the Fig. 4 shows. This plenoptic picture measures $7240 * 5433$. It has $N X=96$ and $N Y=72$. And each sub image has a resolution of $75 * 75$.

We render refocusing images under different rendering aperture $N_{F}$ and refocus factor $\alpha$.

Fig. 5(a) is rendered result under the parameter setting of $P_{i n}=12, N_{F}=4$. The light projection of a point $\left(s_{\alpha}, t_{\alpha}\right)$ on the refocusing plane lays over a diameter of $L=7$ micro lenses. The output result is refocused at the face of the person in the front. We can see that the person in the back is out of focus and has a blurred face. The resolution of output image measures $1152 * 864$.

Fig. 5(b) is rendered result under the parameter setting of $P_{i n}=10, N_{F}=4$. The output result is refocused at the face of the person in the back. And the person in front of him has a blurred face. The resolution of output image measures $920 * 760$. The resolution of rendered result is a function of factor $\alpha$. When we refocus at the further object, we get a lower output resolution.

Fig. 5(c) is rendered result under the parameter setting of $P_{\text {in }}=12, N_{F}=8$. The rendered result is refocused at the face of the person in the front. And it has $L=3$. Comparing Fig. 5(c) with Fig. 5(a), despite that they focus at the same plane, Fig. 5(c) is rendered with a small aperture parameter. So Fig. 5(c) has a extended DOF comparing with Fig. 5(a). As Fig. 5(c) shows, the person in the back is almost within the DOF. His face becomes sharp. But in Fig. 5(a), it is blurred. It can be observed that in Fig. 5(c) the confusion of the background of the scene also decreases.

Fig.5(d) is the result when $N_{F}$ reaches the maximum. The specified rendering aperture is minimal. Under the condition, for each rendering pixel on the refocus plane only one micro lens contributes to it. It has $L=1$. The rendered image is all-in-focus. We can see from Fig. 5(d) that from the two people in the foreground to the wooded area in the background, the objects in the scene are all focused.
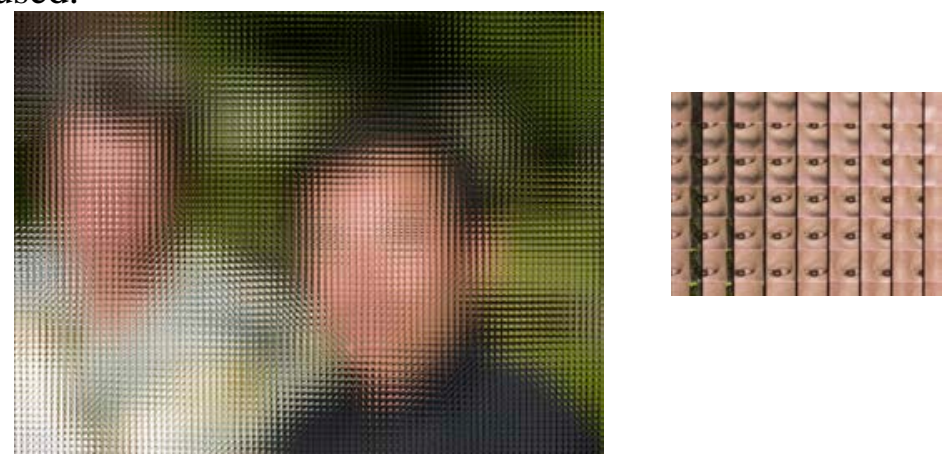

Fig.4. Raw plenoptic data captured by focused plenoptic camera 


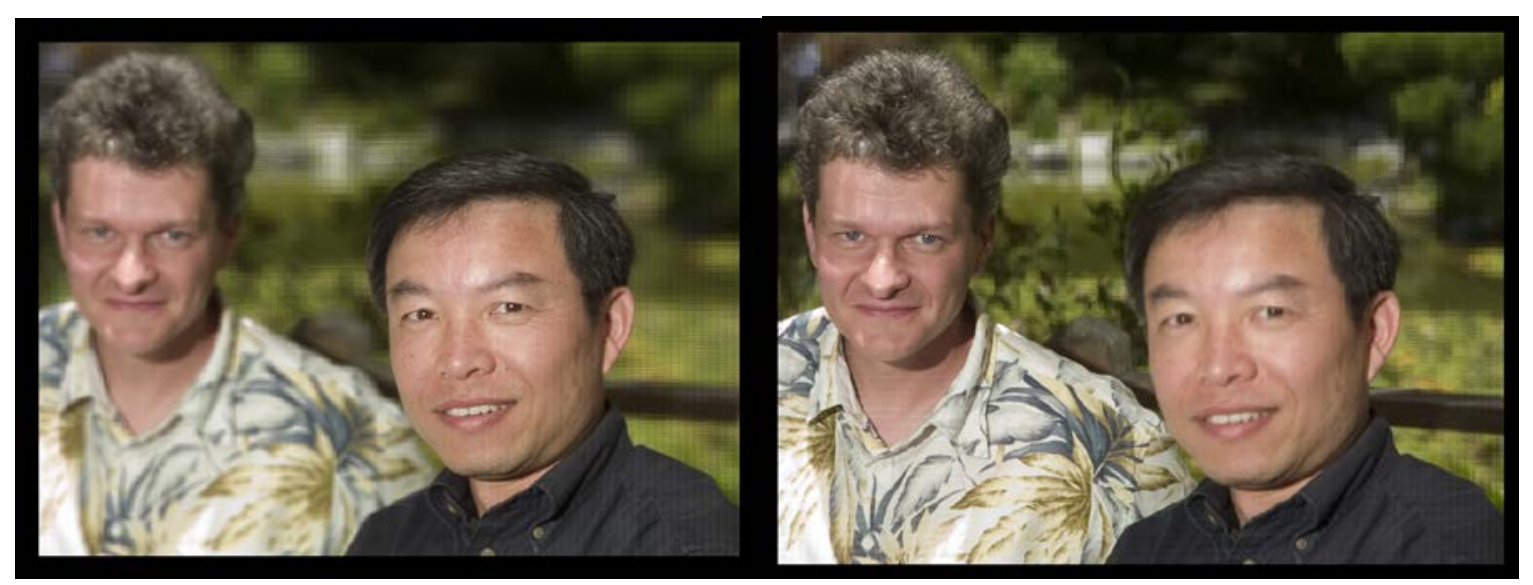

(a) Reconstruction image at $P_{\text {in }}=12, N_{F}=4$

(b) Reconstruction image at $P_{i n}=10, N_{F}=4$

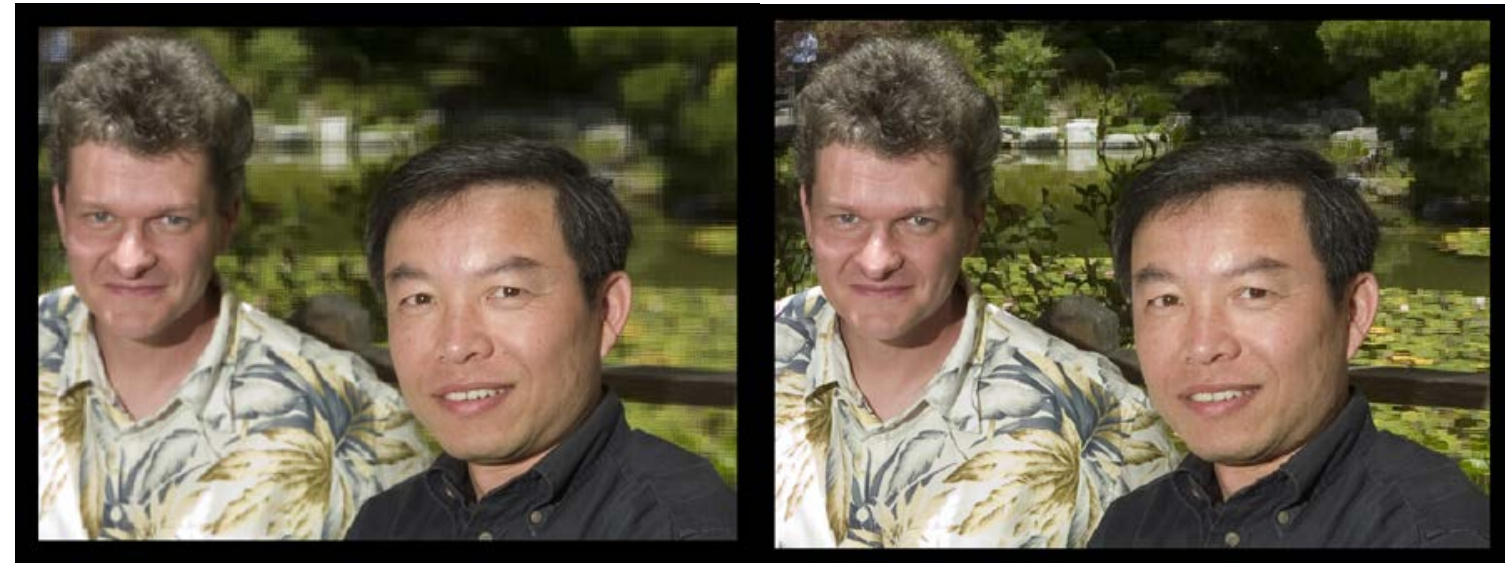

(c) Reconstruction image at $P_{\text {in }}=12, N_{F}=9.6 \quad$ (d) Reconstruction image at $L=1$

Fig.5. The experimental results

\section{Conclusion}

Refocusing after exposure is one application field of the plenoptic camera. Based on the structure of focused plenoptic camera, the paper proposed a digital refocusing algorithm which enables us to refocus the scene at a high output resolution. Besides, by specifying the aperture factor, the algorithm gives us the capability of controlling the DOF of the rendered image. It realizes digital DOF control after exposure and extend the application field of plenoptic camera. Through the rendered result, the algorithm is proved to be feasible and effective.

\section{Acknowledgement}

The research work was supported by State Key Laboratory of Wireless Mobile Communications, China Academy of Telecommunications Technology (CATT).

\section{References}

[1] Adelson,T. and Wang. Single lens stereo with a plenoptic camera. IEEE Trans Pattern Anal Machine Intell, 14:99-106, 1992.

[2] Ng, Levoy M. et al. Light field photography with a hand-held plenoptic camera. CTSR02. California: Stanford University Computer Science. 2005.

[3] Lumsdaine, A. and Georgiev, T. The focused plenoptic camera. International Conference on Computational Photography, April 2009.

[4] Chihchieh Chen, Yichang Lu, Mingshing Su. Light Field Based Digital Refocusing Using a DSLR Camera with a Pinhole Array Mask. ICASSP2010 
[5] A. Lumsdaine. T. Georgiev. Full Resolution Light-field Rendering. Teach.rep, Adobe Systems, January 2008.

[6] A. Lumsdaine. T. Georgiev. Focused Plenoptic Camera and Rendering.

[7] Liu Peng, Liu Dijun. High resolution digital refocusing based on focused plenoptic camera. ICCSA 2014

[8] Liu Peng, Liu Dijun. All-in-focus image reconstruction in plenoptic cameras ICIG 2013 